Sādhanā Vol. 30, Part 1, February 2005, pp. 11-20. @ Printed in India

\title{
Evaluation of LES models for flow over bluff body from engineering application perspective
}

\author{
$S_{\text {VENGADESAN }}{ }^{1}$ and A NAKAYAMA ${ }^{2}$ \\ ${ }^{1}$ Department of Applied Mechanics, Indian Institute of Technology-Madras, \\ Chennai 600 036, India \\ ${ }^{2}$ Department of Science of Regional and Built Environments, Graduate School of \\ Science \& Technology, Kobe University, Kobe 657-8501, Japan \\ e-mail: vengades@iitm.ac.in
}

MS received 7 February 2004; revised 18 June 2004

\begin{abstract}
Three SGS stress closure LES models are evaluated for turbulent flow over a square cylinder. Emphasis is placed on solving engineering-applicationtype problems on affordable computer resources and within reasonable turnaround times. Results are compared with available experimental data and previously published workshop results. Numerical strategies are kept the same for all the cases. Results are also discussed keeping in view limitations of LES methodology of modelling for practical problems and current developments. It is concluded that a one-equation model for subgrid kinetic energy is the best choice.
\end{abstract}

Keywords. Subgrid scale stress models; engineering flows; flow over bluff body.

\section{Introduction}

With the advancement in the power of digital computers, supplemented by developments of accurate and efficient numerical or analytical methods over the past decades, many turbulent flow calculations are reported. Barring developments in Reynolds Averaged NavierStokes (RANS) solution approach, several investigators have noted limitations especially for unsteady flow with this approach (Leschziner 2002; Wilcox 2003). The problem is inherent in the construction of turbulence models itself. There are two approaches to overcome this limitation: Direct numerical simulation (DNS) and large-eddy simulation (LES). In DNS, all the length scales of motions are resolved and the governing equations are solved without any modelling assumptions. Moin \& Mahesh (1998) review developments in the DNS method till then. Owing to high computational requirement, DNS has been limited to simple geometries and for flow at low Reynolds number and its application to engineering-type problems does not appear very likely in the near future.

The large eddy simulation technique is a technique intermediate between the direct simulation and RANS. In this method, large eddies are computed and the smallest, subgrid-scale (SGS) eddies are modeled. This technique which has been thought to be expensive has a high potential to be exploited in various engineering applications. There has been extensive work 
in making this method a useful tool. Ever since Smagorinsky (1963) proposed the first model for the SGS stresses, many newer models have been developed. Piomelli (1999) summarises various SGS models developed till then. Moin (1998) brings out numerical and physical issues in LES, while Rodi (1998) reports that the outcome of workshop on comparative exercise of application of LES models for flow over bluff body as inconclusive. Recently, Morinishi \& Vasilyev (2001) proposes another model with suggestion on the existing dynamic twoparameter mixed SGS model of Salvetti \& Banerjee (1995).

Newer models are demonstrated either for simple flows like channel flow or for a particular type flow that the modeler is directly concerned. Thus their extensions to practical flows with near wall coarse mesh, varying geometrical complexity, involving additional physical and chemical processes are yet to be performed. The performance of the model also depends on how well the near wall features are captured through appropriate boundary conditions. For example, Fureby et al (1997) compared eight SGS models in homogeneous isotropic turbulence and reported that gross features of the flow are virtually insensitive to models. In another study, Vreman et al (1997) reports that of the six models that they considered, mixed dynamic model of Zang et al (1993) was the most successful one for calculation of turbulent mixing layer. While adopting different models, solving additional transport equations and/or calculating stresses by explicit filtering involves some programming efforts and additional computational loads and results depend on type of filtering and grid used.

Though LES method has been beginning to be successful, in order to apply this technique to practical high Reynolds number flows as the case in aerospace and environmental applications there is always a limitation. This is due to extensive demand on the grid to resolve wall layer in order to capture all the energy carrying eddies, which in turn scales in proportion to distance from the wall. This fine resolution on the mesh is to be associated with smaller time step. Spalart (1999) points out that extension of LES approach to aircraft flight Reynolds number for complete aircraft within turn around computation time of one week would not be possible before year 2045. As an alternative, he proposes Detached Eddy Simulation (DES), wherein the near wall flow is solved by RANS approach and the core flow is by LES approach. He also contemplated to use Spalart \& Allmaras (1992) one-equation RANS model for this concept. In this one-equation model, one solves transport equation for working eddy viscosity and expressing the length scale appearing in the equation suitably, this DES approach was demonstrated for channel flow (Nitkin et al 2000). This modelling method inherently had another constant $C_{D E S}$ and the interface where switching off from RANS to LES typically depends on the grid, which is not a desirable situation. This hybrid approach is now being attempted using various other RANS models like Menter SST model (Stretlets 2001), multiscale $k-\varepsilon$ model (Nichols \& Nelson 2003) and low Re $k-\omega$ model (Davidson \& Peng 2003). However, it is easier to couple one-equation SGS model with RANS model due to similar nature of transport equation.

The purpose of this paper is to evaluate three LES models for turbulent flow over a square cylinder from an engineering application perspective. The three models chosen are namely, (i) conventional Smagorinsky model, (ii) dynamic Smagorinsky, and (iii) one-equation model for subgrid kinetic energy. The first two models are widely used. In the third model, one solves a transport equation for subgrid kinetic energy and here the model proposed by Yoshizawa \& Horitui (1985) is considered. It is derived based on two scale direct interaction approximation (TSDIA) theory. All the three models are easy to implement in the code. These models are examined for bluff body flow of typical engineering scale with a realistic grid resolution on affordable computer resources. The grids do not resolve the wall layer at practical Reynolds numbers. This work is a continuation of earlier work (Nakayama \& Vengadesan 2002, 
hereafter referred to as NV), where the influence of grid size, finite difference methods and subgrid-scale stress models for the same geometry were discussed. It was concluded from that study that increasing the order of accuracy or implementing the dynamic procedure improved the results, but they all incur substantially increased computational loads. Additional computations employing one-equation model are considered here. Results are compared and discussed.

\section{Basic equations and description of SGS models considered}

The basic equations used in LES are three-dimensional, time dependent, filtered Navier-Stokes equations. In this study incompressible flow is considered. If the spatially filtered variable of any instantaneous quantity in the $x_{i}$ direction is represented by \langle\rangle , the filtered equation of motion are

$$
\begin{aligned}
\partial\left\langle u_{i}\right\rangle / \partial x_{i}= & \\
\left(\partial\left\langle u_{i}\right\rangle / \partial t\right)+\left(\partial\left\langle u_{i}\right\rangle\left\langle u_{j}\right\rangle / \partial x_{j}\right)= & -\left(\partial\langle p\rangle / \partial x_{i}\right)+v\left(\partial^{2}\left\langle u_{i}\right\rangle / \partial x_{j} \partial x_{j}\right) \\
& -\left(\partial / \partial x_{j}\right) \tau_{i j}^{r},
\end{aligned}
$$

where $\rho$ and $v$ are the fluid density and the kinematic viscosity respectively, and $\tau_{i j}^{r}=$ $-\left\langle u_{i} u_{j}\right\rangle+\left\langle u_{i}\right\rangle\left\langle u_{j}\right\rangle$ is the subgrid turbulence stress. The subgrid residue stress leads to a closure problem and needs to be modeled to obtain numerical solution. The three models considered are described below.

\subsection{Conventional smagorinsky model}

Smagorinsky (1963) proposed the first subgrid-scale stress model. In this model, the residual stress takes the Boussinesq eddy viscosity form

$$
\tau_{i j}^{r}=(2 / 3) k_{s} \delta_{i j}-2 v_{G} S_{i j},
$$

where $S_{i j}$ is the strain tensor of the filtered velocity, $v_{G}$ is the subgrid eddy-viscosity coefficient, $k_{s}$ is the subgrid kinetic energy and $\delta_{i j}$ is the Kronecker delta. Subgrid eddy-viscosity $v_{G}$ and subgrid kinetic energy $k_{s}$ are

$$
v_{G}=\left(C_{S} \Delta\right)^{2}\left(S_{i j} S_{i j}\right)^{1 / 2} \text { and } k_{s}=v_{G}^{2} /\left(C_{k} \Delta\right)^{2} .
$$

Here, $C_{S}$ is the Smagorinsky constant. $C_{k}$ is another model constant. In the present study, numerical values of 0.13 and 0.094 have been set for $C_{S}$ and $C_{k}$ respectively to account appropriately for the energy in the resolvable turbulence (Deardorff 1970). $\Delta$ is the filter width (grid size), and is the characteristic length scale of the largest subgrid-scale eddies and is taken to be the geometric average of the grid spacing in three directions, $\Delta=\left(\Delta x_{1} \Delta x_{2} \Delta x_{3}\right)^{1 / 3}$.

\subsection{Dynamic model}

The major drawback of the Smagorinsky model is the choice of constant $C_{S}$ as there is no standard way of choosing this constant. Ad-hoc adjustments have to be made for different flow type and non-equilibrium flows to account properly for the energy dissipation. With the desire to eliminate these problems, the dynamic model was proposed by Germano et al (1991). In this model, along with Lilly's (1992) modification, $C_{s}$ is determined from

$$
C_{S}^{2}=-\left[\Lambda_{i j} M_{i j}\right] /\left\{2\left[M_{i j} M_{i j}\right]\right\},
$$


where

$$
\begin{aligned}
\Lambda_{i j} & =\left\langle u_{i} u_{j}\right\rangle-\left\langle u_{i}\right\rangle\left\langle u_{j}\right\rangle, \\
M_{i j} & =\widehat{\Delta}^{2}|\widehat{S}| \widehat{S}_{i j}-\Delta^{2}|S| S_{i j},
\end{aligned}
$$

and [ ] once again represents averaging in the spanwise (homogeneous) direction. The caret, ., represents the test filtering. Gaussian test filtering is applied in this study. The ratio of test filter width to that of grid filter is given by the parameter, $\alpha$, which is chosen to be 2 , and as has been done by Lilly (1992), negative values of $C_{s}$ are clipped to avoid unrealistic values of the eddy viscosity.

\subsection{One-equation model}

One-equation for subgrid-scale kinetic energy was proposed by Yoshizawa \& Horiuti (1985). They solve a transport equation for the subgrid-scale kinetic energy, $k_{s}$, to obtain the velocity scale. Thus, this model incorporates history and non-local effects, and this approach is analogous to one-equation models in the RANS methodology. The turbulent transport and pressure diffusion are modelled jointly as an added diffusion, and the viscous dissipation taken to be proportional to $k_{s}^{3 / 2} / \Delta$. The one-equation model obtained is given below,

$$
\begin{aligned}
v_{G} & =C_{\nu} k_{s}^{1 / 2} \Delta, \\
\frac{\partial k_{s}}{\partial t}+\left\langle u_{j}\right\rangle \frac{\partial k_{s}}{\partial x_{j}} & =\frac{\partial}{\partial x_{j}}\left[\left(C_{\nu} k_{s}^{1 / 2} \Delta+v\right) \frac{\partial k_{s}}{\partial x_{j}}\right]-C_{\varepsilon} \frac{k_{s}^{3 / 2}}{\Delta}+2 v_{G} S^{2},
\end{aligned}
$$

where, $S$ is the magnitude of the strain tensor. The terms in (9) are, starting from the left, the time derivative term, convective term, diffusion term, dissipation term and production term. They have also recommended values for constants to be $C_{v}=0.07$ and $C_{\varepsilon}=1.0$ based on the investigation of a channel flow. This model was used by Horiuti (1985) for channel flow to study the budget for subgrid-scale kinetic energy.

\section{Numerical methods}

Calculated flow configuration, coordinate definition and boundary conditions are given in figure 1. This is the same configuration that was chosen by NV. In that study, two grids were chosen, viz. G1 and G2. Grid G1 is about $101 \times 91 \times 21$ points and grid G2 is about $130 \times 111 \times 21$ points. In grid G2, the smallest spacing on the surface is 0.04 times the cylinder side $D$ and total number of grid points is about $3 \cdot 0 \times 10^{5}$, which can be managed by most workstation computers. In the parallel configuration, this grid size requires about ten days of turnaround time for one calculation. Convective terms are discretised by third order upwind biased (UB3) scheme for stability purpose and integrated in time by second order Adams Bashforth scheme. The viscous and the subgrid terms are differenced by the second order central difference, and written in fully implicit form so that time advancing is done using an SOR iterative method. The pressure is solved using the HSMAC (Highly Simplified Marker And Cell) procedure. These procedures are same as those that are described in NV. 


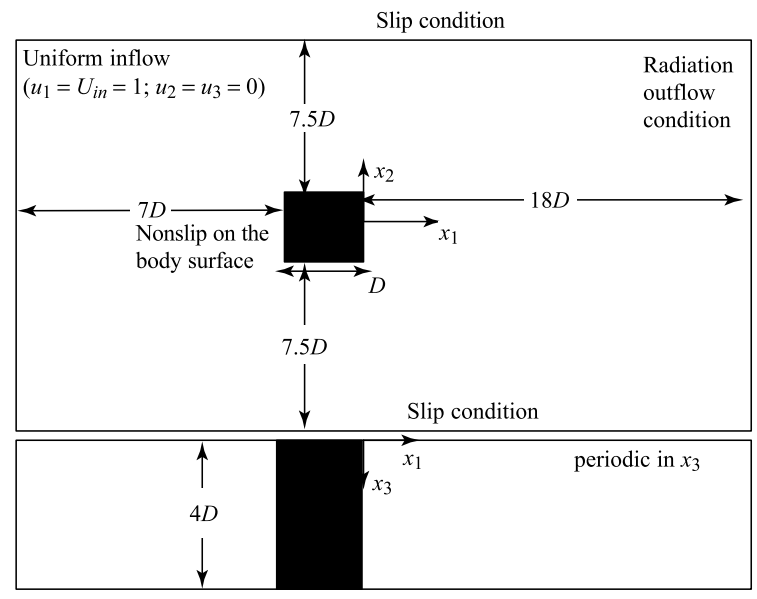

Figure 1. Computational domain and boundary conditions.

\section{Results and discussion}

A standard benchmark test case of turbulent flow over square cylinder at Reynolds number $\operatorname{Re}=22,000$ (Lyn \& Rodi 1994, Lyn et al 1995) is considered for comparison. This test flow experiences impingement on the front side and fixed separation point on the corners. Reynolds number is defined based on the oncoming uniform velocity $\left(U_{i n}\right)$ and size of the square cylinder $(D)$. Nakayama \& Vengadesan (2002) have made detailed investigation on the influence of grid size and different discretization methods and SGS models. As the intention of the present study is to evaluate LES models and only grid G2 and UB3 method of discretisation are chosen in this work. From the previous study (NV) they were found to give the best results. Besides, these strategies are also close to those of a participant of the workshop (TAMU2 of Rodi et al 1997). TAMU2 performed calculation for the same test case employing finite volume and time advancement by Euler scheme. In their study, third-order upwind scheme for convective terms was used and statistical averages over 8 vortex shedding cycles were reported. Calculation results with conventional Smagorinsky model, dynamic Smagorinsky model and without any subgrid model with the same numerical strategy that are reported earlier in NV are included here for completeness and comparison. Additional calculations with the one-equation model are performed and results are discussed. The results are obtained after averaging over eight vortex shedding cycles after an initial development time of $50 \mathrm{D} / U_{\text {in }}$.

Though computation without any model is like performing DNS, for the present Reynolds number with chosen mesh size, since all the scales of motion up to the Kolmogrov length scale are not resolved, present computation is not qualified to be called DNS. Both from table 1 and subsequent comparison graphs, one can observe that prediction by this procedure is not good. Comparison of mean and the RMS fluctuations of the lift and drag coefficients, maximum back flow, Strouhal number along with those by experiment and TAMU2 are given in table 1 . The mean lift coefficient predicted by one-equation model is slightly lesser. As predictions of mean velocity (figure 2a) and pressure distribution on the surface cylinder (figure 3 ) by both LES models fall close to each other, all other bulk quantities predicted by the one-equation model are comparable to those by the dynamic LES model. It means that as long as one does not resolve close to wall and capture near-wall physical phenomena well, refinements in the model do not bring any improvement in the mean parameters. 


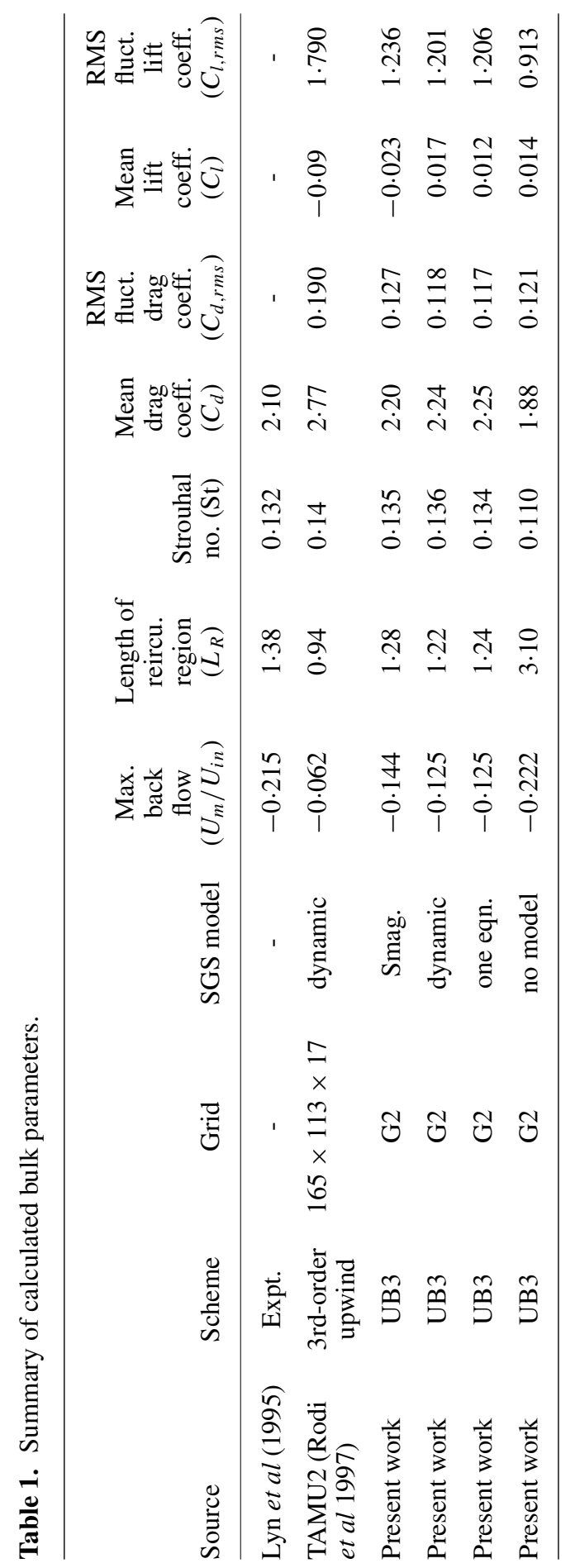



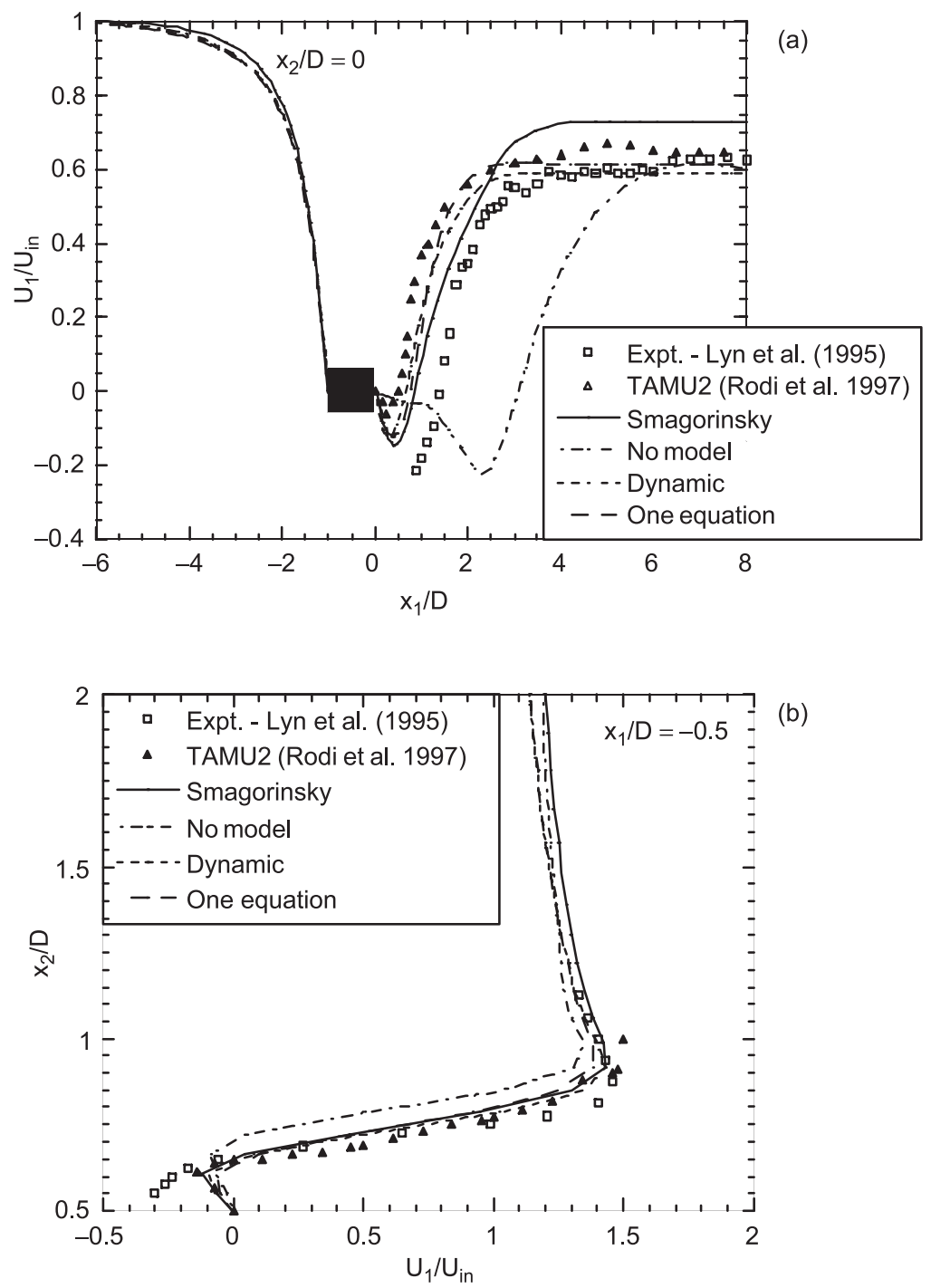

Figure 2. Comparison of the streamwise component of mean velocity (a) along the wake centreline, (b) on the cylinder side, $x_{1} / D=-0.5$.

Figures $2 \mathrm{a}$ and $\mathrm{b}$ show the distribution of the time-averaged streamwise velocity component $\left(U_{1} / U_{i n}\right)$ along the wake centerline and on the cylinder side surface $\left(x_{1} / D=-0 \cdot 5\right)$. These quantities are compared with those of Lyn's experiment (Lyn 1994, 1995) and one of the results, TAMU2 reported at the workshop. When compared with dynamic procedure, oneequation model captures the recovery of the velocity in the wake region and back flow well. All the models perform almost similarly when compared for mean velocity distribution on the cylinder side surface. This probably can be attributed to inadequate resolution of boundary layer with the present mesh size for the moderate Reynolds number that is considered. This will be the situation for simulation of most engineering type practical flow problems on affordable 


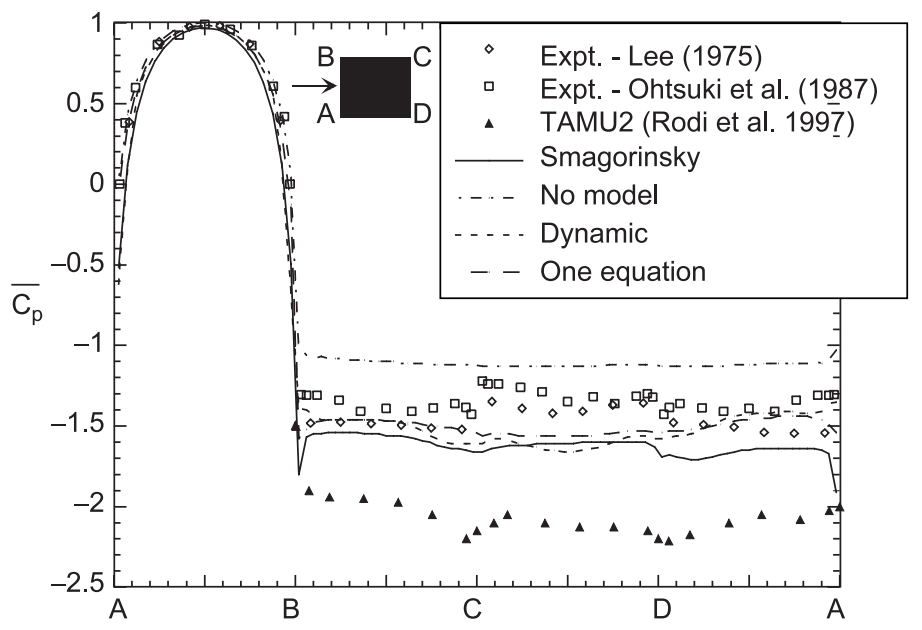

Figure 3. Comparison of surface pressure distribution.

computer resources or scenario when the solution is expected within reasonable turnaround time. Surface pressure distribution along with those by Lee (1975) and Ohtsuki et al (1987) are compared in figure 3 . There is an improvement in going from simpler model to either dynamic model or one-equation model. Comparison of prediction of turbulence stresses are shown in figures $4 \mathrm{a}$ and $\mathrm{b}$. As observed in surface pressure distribution, there is improvement in the prediction due to refinement in the model. The peak is captured and stress in the wake region is also reasonably reproduced.

When compared to predictions without a SGS model and by a conventional Smagorinsky model, performances by the dynamic model and the one-equation model show that improvements and results by both models are comparable to each other. The refinements in the models probably represent sub-grid residue stresses better and non-local effects are accounted for appropriately. Though the one-equation model requirement is slightly on the lower side, both the models require additional computational time, when compared to that for the standard Smagorinsky model. As explained in the introduction, to address the problem of near-wall mesh resolution, from the hybrid LES perspective, though results by both considered refined LES models are comparable, the one-equation LES model is found to be the better choice as this is easier to couple with the RANS model.

\section{Conclusion}

Three SGS LES models, viz standard Smagorinsky model, dynamic model and one-equation model for subgrid kinetic energy, are evaluated from the engineering application perspective. Turbulent flow past a square cylinder, which is a standard benchmark test case problem, is considered for the present study. Moderately high Reynolds number and solution with grid size, manageable on affordable computational resources, are the main emphasis here. Results are compared and discussed in-line with alternative developments on the modelling side. It is inferred that the one-equation SGS model meets the requirement. 

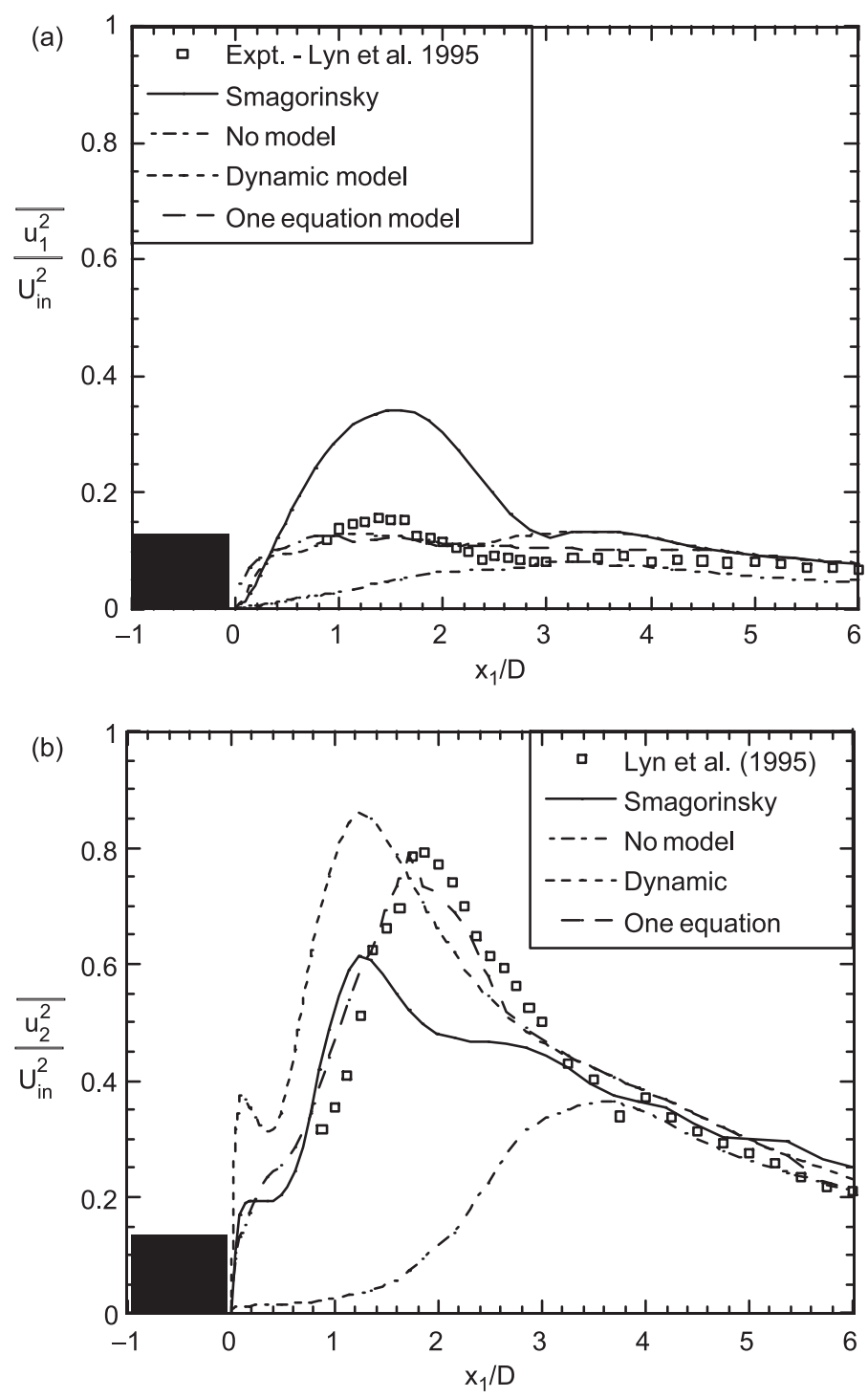

Figure 4. Comparison of turbulent stresses, (a) streamwise component and (b) transverse component along the wake centreline.

\section{References}

Deardorff J W 1970 A numerical study of three-dimensional turbulent channel flow at large Reynolds numbers. J. Fluid Mech. 41: 453-480

Davidson L, Peng S H 2003 Hybrid LES-RANS modelling: a one-equation SGS model combined with a $k-\omega$ model for predicting recirculating flows. Int. J. Numer. Methods Fluids 43: 1003-1018 Fureby C, Tabor G, Weller W G, Gosman A D 2000 A comparative study of subgrid scale models in homogeneous isotropic turbulence. Phys. Fluids 9: 1416-1429

Germano M, Piomelli U, Moin P, Cabot W H 1991 A dynamic subgrid scale eddy viscosity model. Phys. Fluids A3: 1760-1765 
Horiuti K 1985 Large eddy simulation of turbulent channel flow by one-equation modelling. J. Phys. Soc. Jap. 54: 2855-2865

Leschziner M A 2002 At the crossroads of turbulence modelling and simulation: Opportunities and challenges. Advances in fluid modelling and turbulence measurements. (Singapore: World Scientific) pp 3-19

Lee B E 1975 The effects of turbulence on the surface field of a square prism. J. Fluid Mech. 69: 263-282

Lilly D K 1992 A proposed modification of the Germano subgrid-scale closure method. Phys. Fluids A4: 633-635

Lyn D A, Rodi W 1994 The flapping shear layer formed by flow separation from the forward corner of a square cylinder. J. Fluid Mech. 267: 353-376

Lyn D A, Einav S, Rodi W, Park J H 1995 A laser Doppler velocimetry study of ensemble-averaged characteristics of the turbulent flow near wake of a square cylinder. J. Fluid Mech. 304: 285-319

Moin P 1998 Numerical and physical issues in large eddy simulation of turbulent flows. JSME Int. J. B41: 454-463

Moin P, Mahesh K 1998 Direct numerical simulation - A tool in turbulence research. Annu. Rev. Fluid Mech. 30: 539-578

Morinishi Y, Vasilyev O V 2001 A recommended modification to the dynamic two-parameter mixed subgrid scale model for large eddy simulation of wall bounded turbulent flow. Phys. Fluids A13: $3400-3410$

Nakayama A, Vengadesan S N 2002 On the influence of numerical schemes and subgrid-stress models on large eddy simulation of turbulent flow past a square cylinder. Int. J. Numer. Methods Fluids 38: 227-253

Nichols R H, Nelson C C 2003 Application of hybrid RANS/LES turbulence models. 41st Aerospace Science Meetings and Exhibit, AIAA 2003-0083

Nitkin N V, Nicoud F, Wasistho B, Squires K D, Spalart P R 2000 An approach to wall modelling in large-eddy simulations. Phys. Fluids A12: 1760-1765

Ohtsuki H, Fujii K, Washizu H, Ohya A 1987 On the characteristics of three components of aerodynamic forces and pressure distribution around a fixed rectangular cylinder in a uniform flow. 5th Symp. Wind Loads on Structures, pp. 169-175

Piomelli 1999 Large-eddy simulation: achievements and challenges. Prog. Aerosp. Sci. 35: 335-362

Rodi W, Ferziger J H, Breuer M, Pourquie M 1997 Status of large eddy simulation: Results of a Workshop. ASME J. Fluids Eng. 119: 248-262

Rodi W 1998 Large-eddy simulations of the flow past bluff bodies: State-of-the-art. JSME Int. J. B41: 361-374

Salvetti M, Banerjee S 1995 A priori tests of a new dynamic subgrid-scale model for finite difference large-eddy simulations. Phys. Fluids A7: 2831-2837

Smagorinsky J 1963 General circulation experiments with primitive equations - I, The basic experiment. Mon. Weather Rev. 91: 99-165

Spalart P R 1999 Strategies for turbulence modelling and simulations. Engineering turbulence modelling and experiments - 4 (New York: Elsevier Science)

Spalart P R, Allmaras S R 1992 A one-equation turbulence model for aerodynamic flows. AIAA Paper - 92-0439

Strelets M 2001 Detached eddy-simulation of massively separated flows. 39th Aerospace Sciences Meeting and Exhibit AIAA 2001-0879

Vreman B, Geurts B, Kuerten K 1997 Large-eddy simulation of turbulent mixing layer. J. Fluid Mech. 339: $357-390$

Wilcox D C 2003 Turbulence modelling for CFD 3rd edn. (Los Angeles, CA: DCW Industries)

Yoshizawa A, Horiuti K 1985 A statistically-derived subgrid-scale kinetic energy model for the largeeddy simulation of turbulent flows. J. Phys. Soc. Jap. 54: 2834-2839

Zang Y, Street R L, Koseff J R 1993 A dynamic mixed subgrid-scale model and its application to turbulent recirculating flows. Phys. Fluids A5: 3186-3196 